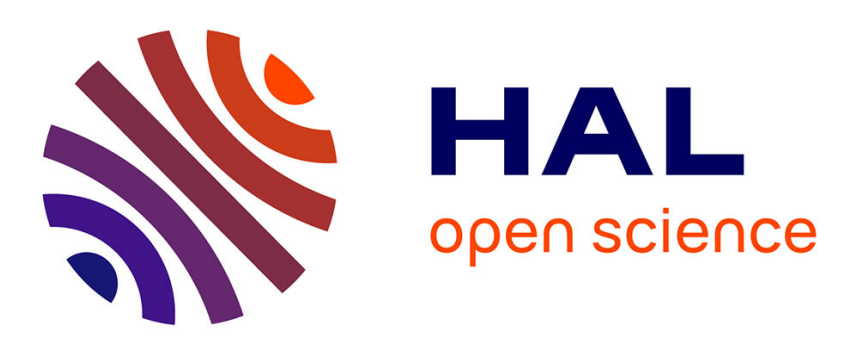

\title{
Coupling predictive scheduling and reactive control in manufacturing: state of the art and future challenges
}

Olivier Cardin, Damien Trentesaux, André Thomas, Pierre Castagna, Thierry Berger, Hind Bril

\section{To cite this version:}

Olivier Cardin, Damien Trentesaux, André Thomas, Pierre Castagna, Thierry Berger, et al.. Coupling predictive scheduling and reactive control in manufacturing: state of the art and future challenges. 4th International Workshop on Service Orientation in Holonic and Multi-Agent Manufacturing, SOHOMA 2014, Nov 2014, Nancy, France. hal-01094788

\section{HAL Id: hal-01094788 \\ https://hal.science/hal-01094788}

Submitted on 15 Dec 2014

HAL is a multi-disciplinary open access archive for the deposit and dissemination of scientific research documents, whether they are published or not. The documents may come from teaching and research institutions in France or abroad, or from public or private research centers.
L'archive ouverte pluridisciplinaire HAL, est destinée au dépôt et à la diffusion de documents scientifiques de niveau recherche, publiés ou non, émanant des établissements d'enseignement et de recherche français ou étrangers, des laboratoires publics ou privés. 


\title{
Coupling predictive scheduling and reactive control in manufacturing: state of the art and future challenges
}

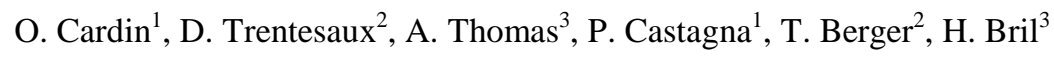 \\ ${ }^{1}$ LUNAM Université, IUT de Nantes - Université de Nantes, IRCCyN UMR CNRS 6597 (Ins- \\ titut de Recherche en Communications et Cybernétique de Nantes), 2 avenue du Prof. Jean \\ Rouxel - 44475 Carquefou \\ Olivier.Cardin@univ-nantes.fr \\ Pierre.Castagna@univ-nantes.fr \\ ${ }^{2}$ Université Lille Nord de France, UVHC, Tempo-Lab., F-59313 Valenciennes France \\ Damien.Trentesaux@univ-valenciennes.fr \\ Thierry.Berger@univ-valenciennes.fr \\ ${ }^{3}$ Research Centre for Automatic Control (CRAN), CNRS (UMR 7029), Nancy University, \\ ENSTIB, 27 rue Philippe Seguin, 88000 Epinal, France \\ Andre. Thomas@univ-lorraine.fr \\ Hind.Brile univ-lorraine.fr
}

\begin{abstract}
Nowadays, industrials are seeking for models and methods that are not only able to provide efficient overall production performance, but also reactive facing a growing set of unpredicted events. One important research activity in that field focuses on holonic/multi-agent control systems that couple predictive/proactive and reactive mechanisms into agents/holons. Meanwhile, not enough attention is paid to the optimization of this coupling. The aim of this paper is to depict the main research challenges that are to be addressed before expecting a large industrial dissemination. Relying on an extensive review of the state of the art, three main challenges are highlighted: the estimation of the future performances of the system in reactive mode, the design of efficient switching strategies between predictive and reactive modes and the design of efficient synchronization mechanisms to switch back to predictive mode.
\end{abstract}

Keywords: holonic control, predictive/reactive, performance indicator, discrete-event observer, flexible manufacturing system.

\section{Introduction}

Classical (historical) predictive approaches consist in using a centralized predictive scheduling system loosely coupled with a reactive control system that implements it. The scheduling models are based on a mathematical representation of the production system from which an optimization or heuristic algorithm is designed and computed in a centralized way. This approach leads to determine or approximate the optimal sequence of tasks to be executed in the system in order to maximize one or several criterion(s) somehow related to productivity, customer satisfaction, etc. The result of

adfa, p. 1, 2011.

(C) Springer-Verlag Berlin Heidelberg 2011 
the calculation is then used by the Manufacturing Execution System (MES) for the Production Activity Control of the production system[1]. This approach is considered optimal as long as the modeling of the production system is realistic but also deterministic. In such an approach, parameters are simplified in order to fasten up the calculations. If stochastic changes of parameters are significant (e.g. duration of manual operations, breakdowns or failures), the execution of the schedule in the production system gives results that are generally far from optimal or even inapplicable[2]. Traditionally, the production system is halted at the time when a disruption is detected during the execution of the scheduler then waits for a new schedule to be generated. If the rescheduling phase is long or if disruptions happen frequently, the duration of the rescheduling phase may lead to a drastic reduction of the overall performance. As a consequence, this approach, despite the fact that it has been widely used for several years in a number of industries, cannot be considered as sufficiently efficient nowadays since reactivity issues grow more and more important. Since few years, a research field, dealing with proactive scheduling has emerge. The main idea is to increase the robustness of the predictive schedule, and as a consequence, to limit the "nervousness" of the scheduling/rescheduling iterations, see for example [3]. These technics typically use redundancy (temporal or resource-oriented), probabilistic methods, contingent methods, or objective functions that integrate robustness criteria evaluating the risk to not respect a candidate scheduling given possible perturbations. A growing activity from operation research has emerged in the last few years in that field [4].

Reactive approaches consider every event in real time, with no anticipation. Several approaches can be identified depending on the fact that they are centralized or distributed. When centralized, priority rules (e.g. heuristics-based) are defined and used on the fly, that is, whenever a decision must be taken. The choice of the rule to apply can also be decided dynamically. When distributed, control decisions are distributed among a set of cooperative control entities, being agents or holons, with no hierarchical relationships among them. Distributed approaches have been studied by researchers massively in the 90's, see for example [5], one of the historical reference in this field. These approaches are known to generate applicable solution since decisions are taker according to the real state of the production system. Despite this, they are also known to have their performances rapidly decreasing with time compared to pure predictive ones if no perturbation occurs.

Due to the limitations of these two historical approaches facing the current industrial needs, researchers are more and more considering a last kind of approach by trying to propose scheduling and control architectures and models that couple local reactive mechanisms implemented into agents/holons with global predictive mechanisms, being robust or not. In such predictive/reactive or proactive/reactive approaches (denoted hybrid approaches in the remaining of this paper), some of these control holons/agents are typically interfaced with the predictive scheduling system that provides them with an optimal or approximated scheduling and at the same time, interfaced with components like physical products or resources of the physical production system to control them [6] [7]. 
In such hybrid architectures, the fundamental decision facing perturbation is whether to still follow the predictive/proactive schedule (predictive mode) or not. If not, they may switch to a reactive mode where events and decision are handled in real time with the intention to switch back to a predictive mode as soon as possible. The main issue for researchers is then to provide accurate mechanisms to define the best switching dates (and/or the best switching decision-making levels) for control holons/agents so that they behave in a sense that the whole behavior of the hybrid architecture stay globally optimized despite disturbances. This predictive/proactivereactive coupling issue is not easy to solve: for example, if a broken machine can be repaired quickly, then it may not be necessary for its control holon/agent to switch in reactive mode if the pre-determined schedule will be still accurate because of some slack in the original schedule. Another issue is related to the possible nervousness of the architecture that may often switch from one mode to another [8].

From our point of view, this global issue can be broken down into the following scientific challenges. First, it is necessary to provide tools that enable the estimation of future performances, including disturbance detection, diagnosis and prognostic mechanisms (i.e. evaluation of the impact of a disturbance on the global performances). Second, based on these estimators, it is necessary to design efficient synchronization mechanisms, leading typically to the design of a proper indicator to determine if necessary when it is pertinent to switch back to predictive mode. Third, efficient switching strategies based on these synchronization mechanisms must be designed. These strategies must lead to a fair use of reactive modes (sufficiently to absorb uncertainties, but used as less as possible to avoid decreasing the performance). These strategies must be integrated into a control system considering balanced articulation between hierarchical mechanisms and heterarchical ones, while avoiding nervousness. The following sections propose a literature review structured following these three underlined challenges in the context of hybrid architectures.

\section{First challenge: estimation of future performances}

One fundamental reason explaining the lack of studies devoted to the predictive/proactive-reactive coupling issue in hybrid architectures, is related to the difficulty for researchers to design models enabling them to estimate future performances because of the difficulty to accurately observe the real state of the production system (eg., locate products and their state), and extrapolate possible evolution scenarios in the near future. This feature is mandatory in order to detect at which moment the control should switch from a predictive to a reactive mode. This detection might only be based on prediction models, split into two classes: analytic models, rapidly limited by the size of the considered systems because of their algorithmic complexity, and discrete-event simulation models, able to handle large systems but extremely timeconsuming. This last characteristic often limits their use in the context of real-time decision making.

To solve this issue, an observer able to detect abnormal behavior (difference between theoretical expected behavior and observed behavior - state reconstructor abili- 
ties) and to evaluate the impact of this difference on the global behavior of the system (diagnoser abilities) must be designed [9]. For example, if it is obviously necessary to detect the delay in execution of a task from the predictive schedule, some of these delays might not be critical for the behavior of the system, either because they are very short, or thanks to the available free margin. Many modelling formalisms are classically used to build diagnosers, including automata [10] and their timed and probabilistic extensions, Petri nets [11][12], statecharts and hierarchical state machines [13]. The most promising perspective here would be to implement the diagnoser using online simulation, which is an efficient but hard to implement forecasting tool. These programs are usually dedicated to the dimensioning phase (offline), but are increasingly used as actual systems control tools, included in the control loop (online) [14].

\section{Second challenge: designing efficient synchronization mechanisms}

The "switch down" mechanism consists in switching from a predictive mode where a predictive scheduling is to be executed by holons to a reactive mode where real-time holon decision may override these predictive scheduling decisions. This kind of switching is widely addressed in the literature (event-driven or thresholddriven switch), see for example [7]. But a first issue appears: researchers do not really pay attention to the real need to "switch down" (e.g. if a machine breakdown is shortly repaired, then slack may be used to avoid real-time overriding decisions).

In addition, the "switch back" mechanism that concerns the way the predictive mode is reused after and instead of the reactive mode is rarely addressed or even mentioned. All these decisions must be taken according to global performances objectives targeted by the production manager. To correctly address this challenge, two questions relevant to synchronization have first to be answered to:

- What are the most pertinent criteria to switch down or back?

- How to reinsert these concerned in-progress products in the remaining of the material flow (switch down case), or how to synchronize the new re-optimized schedule with the state of the manufacturing system after this optimized schedule is obtained (switch back case)?

The first question is relative to performance indicators system leading to be able to estimate when it is pertinent to switch down or back according to the circumstances (the physical context: flexible manufacturing system, shop floor, constraints, management rules, etc...). It is obvious that objectives and performance indicators must be determined according to the industrial context and it seems difficult to design generic indicators useful (and applicable) to a particular system. They have probably to be design according to the physical context or at least according to an industrial system class.

Indicator system according to the class of physical context and built on a learning system might be pertinent. Multicriteria optimization based on Choquet integrals 
could, according to the estimators obtained thanks to the first challenge, lead to establish switching points according to measured drifts and situations. This approach is close to the one proposed by [15]. The authors proposed an integrated approach for the automatic design of flexible manufacturing systems using simulation and multicriteria decision-making techniques. In this work, the selection of the most suitable design, based on a multi-criteria decision-making technique, the Analytic Hierarchy Process AHP, is employed to analyze the output from the flexible manufacturing system simulation models. Intelligent tools such as expert systems, fuzzy systems and neural networks, were developed to support the design process of the flexible manufacturing system.

E. Muhl proposed for the automotive industry a way to optimize, in a centralized way, the schedule of the car assembly line according to a unique performance indicator and the determination of the pertinent parameters which were periodically recalculated to assure the best synchronization between the real shop-floor state and the new schedule [16]. Another way to design this indicator system could be found thanks to learning mechanisms as neural networks, fuzzy approaches or Choquet integrals usage [17]. During his PhD work, C. Herrera first merged the two centralized and distributed approaches applied to a similar industrial case [6], [18]. He proposed a multilevel parametric model to solve this re-scheduling problem. But the performance indicator leading to the switch decision has been taken as a hypothesis and the distributed decisions were limited to the first choice with a simple splitting decision to reinsert the remaining parts in the existing predictive schedule. Another research work focusing on the synchronization problem was done by $\mathrm{H}$. El Haouzi [19]. She proposed an original architecture to control manufacturing flows on two assembly lines. In case of disturbances, products can arrive early or late at the synchronization point between the main assembly line and its feeders. The architecture was composed of an ERP and a distributed decision system. The on-line information was provided by Auto-ID technologies.

\section{Third challenge: designing efficient switching strategies integrated into a hybrid control architecture}

Several European projects addressed the designing of distributed/hybrid control architectures into the so-called "smart factories". PABADIS and PABADIS PROMISE are amongst the firsts EU projects in that direction. More recently, let's mention GRACE, SMARTPRODUCT and ARUM projects ${ }^{1}$. The GRACE project is in line with the current need to build modular, intelligent and distributed manufacturing control systems and studied more precisely the impact of manufacturing operation on quality. The distributed control architecture is interfaced with a Manufacturing Execution System (MES). The SMARTPRODUCT project focused the work on the embedding of "proactive knowledge" into smart products. "Proactive" Smart products "talk", "guide", and "assist" designers, workers and consumers dealing with them. Some

1 http://grace-project.org/, http://www.smartproducts-project.eu/ and http://arum-project.eu/ 
proactive knowledge will be co-constructed with the product, while other parts are gathered during the product lifecycle using embedded sensing and communication. Neither GRACE nor SMARTPRODUCT addressed the optimization of the control architecture, being hybrid or not. More recently, an interesting initiative, the ARUM project, aimed at designing a holonic multi-agent system combined with a service architecture designed to improve performance and scalability beyond the state of the art. The proposed solution integrates multiple layers of sensors, legacy systems and agent-based tools for beneficial services like learning, quality, and risk and cost management, including ecological footprints aspects.

In the scientific literature, there also exist different hybrid scheduling and control architectures. [7] pointed out that the main idea is to take advantages of two basic structuration mechanisms: hierarchical (vertical relationships, toward centralization of information and decisions) and heterarchical (horizontal relationships, towards distribution of information and decisions) mechanisms. By doing this, it is expected to avoid their respective drawback (typically: lack of reactivity for hierarchies and myopia for heterarchies). Thus, usually, the hierarchical part of the architecture is responsible for the predictive and global optimization, while the heterarchical part allows reactivity and local optimization. Famous flagship hybrid architectures are PROSA[21], ADACOR [20] or D-MAS [22]. Such hybrid architectures are composed of cooperative decisional control entities, typically modeled as holons or agents. In [7] was proposed an original literature review of several hybrid architectures. Indeed, there are numerous ways to combine the introduced structuration mechanisms. The authors identified two lines of study. The first "structure dynamics" concerns the possibility of the control structure to evolve with time (dynamic structure, e.g. full change from pure hierarchical to a pure heterarchical architecture) or not (static structure). The second "control homogeneity" deals with the way the control is applied: in the same way for every decisional agent/holon (homogeneous control) or not (heterogeneous control). In terms of structure dynamics and homogeneity control, hybrid architectures have thus been positioned according four sub-classes.

Non static hybrid architectures are very promising since they provide (self-) adaptation mechanisms needed to improve the agility of the control system [8]. In such architectures, an important mechanism of switching is responsible to switch holons/agents from/to predictive to/from reactive modes (for a holon/agent or a group of holons/agents). This mechanism adapts dynamically the structure of the control architecture to the production uncertainties in ensuring the performance. Of course, more generally, there may be different intermediary levels and mode between a fully predictive and a fully reactive mode. As a consequence, attention must be paid when designing and optimizing hybrid architectures. Some first ideas have been proposed [7], but they were provided aside the main topic of the referenced paper. Thus, this initial work was clearly insufficient and not really formalized in a generic and effective way. For example, the production order set was assumed to be provided as a whole, in a static manner, with no "on the fly" orders. Under that condition, the switching down was made only once, and with few attention paid to the real need to switch down. Moreover, the switch back was made only at the end of the production of the order set. 
This challenge is complex to address and despite the growing number hybrid architectures proposed in the literature, the way prediction and reaction are coupled is neither optimized nor even clearly justified. This contributes clearly to a lack of applications of such contributions in real situations in industries despite the fact that they respond to a real industrial need. As an illustration, to the best of our knowledge, only P2000+ [23] was applied in Daimler but it failed because of issues related to the proposed research topic (and others issues, such as global cost).

\section{$5 \quad$ Conclusion and perspectives}

This paper depicted a state of the art of predictive-reactive control architectures of manufacturing systems. Even though these hybrid architectures show promising performances on academic examples, three main challenges are still to be investigated from the authors' perspective. Several leads are given to orient future research activities in this field, with the objective of making these concepts applicable on industrial shop floors in the next few years.

\section{References}

[1] D. Trentesaux, C. Pach, A. Bekrar, Y. Sallez, T. Berger, T. Bonte, P. Leitão, and J. Barbosa, "Benchmarking flexible job-shop scheduling and control systems," Control Eng. Pract., vol. 21, no. 9, pp. 1204-1225, Sep. 2013.

[2] O. Cardin, N. Mebarki, and G. Pinot, "A study of the robustness of the group scheduling method using an emulation of a complex FMS," Int. J. Prod. Econ., vol. 146, no. 1, pp. 199-207, Nov. 2013.

[3] T. Chaari, S. Chaabane, T. Loukil, and D. Trentesaux, "A genetic algorithm for robust hybrid flow shop scheduling," Int. J. Comput. Integr. Manuf., vol. 24, no. 9, pp. 821-833, Sep. 2011.

[4] F. Ghezail, H. Pierreval, and S. Hajri-Gabouj, "Analysis of robustness in proactive scheduling: A graphical approach," Comput. Ind. Eng., vol. 58, no. 2, pp. 193-198, Mar. 2010.

[5] V. V. Prabhu and N. A. Duffie, "Modelling and Analysis of Heterarchical Manufacturing Systems Using Discontinuous Differential Equations," CIRP Ann. - Manuf. Technol., vol. 45, no. 1, pp. 445-448, Jan. 1996.

[6] A. Thomas, H. El Haouzi, T. Klein, S. Belmokhtar, and C. Herrera, "Architecture de systèmes contrôlés par le produit pour un environnement de juste à temps," J. Eur. Systèmes Autom., vol. 43, no. 4-5, pp. 513-535, 2009.

[7] C. Pach, T. Berger, T. Bonte, and D. Trentesaux, "ORCA-FMS: a dynamic architecture for the optimized and reactive control of flexible manufacturing scheduling," Comput. Ind., vol. 65, no. 4, pp. 706-720, May 2014.

[8] J. Barbosa, P. Leitão, E. Adam, and D. Trentesaux, "Nervousness in Dynamic Self-organized Holonic Multi-agent Systems," in Highlights on Practical Applications of Agents and Multi-Agent Systems, J. B. Pérez, M. A. Sánchez, P. Mathieu, J. M. C. Rodríguez, E. Adam, A. Ortega, M. N. Moreno, E. Navarro, 
B. Hirsch, H. Lopes-Cardoso, and V. Julián, Eds. Springer Berlin Heidelberg, 2012, pp. 9-17.

[9] J. Zaytoon and S. Lafortune, "Overview of fault diagnosis methods for Discrete Event Systems," Annu. Rev. Control, vol. 37, no. 2, pp. 308-320, Dec. 2013.

[10] M. Sampath, R. Sengupta, S. Lafortune, K. Sinnamohideen, and D. Teneketzis, "Diagnosability of discrete-event systems," IEEE Trans. Autom. Control, vol. 40, no. 9, pp. 1555-1575, Sep. 1995.

[11] M. P. Cabasino, A. Giua, and C. Seatzu, "Fault detection for discrete event systems using Petri nets with unobservable transitions," Automatica, vol. 46, no. 9, pp. 1531-1539, Sep. 2010.

[12] M. Dotoli, M. Pia Fanti, A. M. Mangini, and W. Ukovich, "Identification of the unobservable behaviour of industrial automation systems by Petri nets," Control Eng. Pract., vol. 19, no. 9, pp. 958-966, Sep. 2011.

[13] A. Paoli and S. Lafortune, "Diagnosability Analysis of a Class of Hierarchical State Machines," Discrete Event Dyn. Syst., vol. 18, no. 3, pp. 385-413, Sep. 2008.

[14] O. Cardin and P. Castagna, "Using online simulation in Holonic manufacturing systems," Eng. Appl. Artif. Intell., vol. 22, no. 7, pp. 1025-1033, Oct. 2009.

[15] F. T. S. Chan, B. Jiang, and N. K. H. Tang, "The development of intelligent decision support tools to aid the design of flexible manufacturing systems," Int. J. Prod. Econ., vol. 65, no. 1, pp. 73-84, Apr. 2000.

[16] E. Muhl, P. Charpentier, and F. Chaxel, "Optimization of physical flows in an automotive manufacturing plant: some experiments and issues," Eng. Appl. Artif. Intell., vol. 16, no. 4, pp. 293-305, Jun. 2003.

[17] P. Thomas and A. Thomas, "Multilayer perceptron for simulation models reduction: Application to a sawmill workshop," Eng. Appl. Artif. Intell., vol. 24, no. 4, pp. 646-657, Jun. 2011.

[18] C. Herrera, "Cadre générique de planification logistique dans un contexte de décisions centralisées et distribuées," Université Henri Poincaré - Nancy I, 2011.

[19] H. El Haouzi, J.-F. Pétin, and A. Thomas, "Design and validation of a productdriven control system based on a six sigma methodology and discrete event simulation," Prod. Plan. Control, vol. 20, no. 6, pp. 510-524, 2009.

[20] P. Leitao, A. W. Colombo, and F. J. Restivo, "ADACOR: A Collaborative Production Automation and Control Architecture," IEEE Intelligent Systems, vol. 20, no. 1, pp. 58-66, 2005.

[21] H. Van Brussel, J. Wyns, P. Valckenaers, L. Bongaerts, and P. Peeters, "Reference architecture for holonic manufacturing systems: PROSA," Comput. Ind., vol. 37, no. 3, pp. 255-274, Nov. 1998.

[22] P. Verstraete, B. S. Germain, P. Valckenaers, H. V. Brussel, J. V. Belle, and Hadeli, "Engineering Manufacturing Control Systems Using PROSA and Delegate MAS," Int J Agent-Oriented Softw Eng, vol. 2, no. 1, pp. 62-89, Jan. 2008.

[23] S. Bussmann and K. Schild, "An agent-based approach to the control of flexible production systems,” 2001, vol. 2, pp. 481-488. 